\title{
Tipping point analysis of cracking in reinforced concrete
}

\author{
M. Perry ${ }^{1}$, V. Livina ${ }^{2}$, P. Niewczas ${ }^{3}$ \\ ${ }^{1}$ Department of Civil and Environmental Engineering, James Weir Building, \\ University of Strathclyde, Glasgow, G1 1XJ, UK \\ 2 National Physical Laboratory, Hampton Road, Teddington, TW11 0LW, UK \\ ${ }^{3}$ Department of Electronic and Electrical Engineering, Royal College Building, \\ University of Strathclyde, Glasgow, G1 1XW, UK \\ E-mail: m.perry@strath.ac.uk
}

\begin{abstract}
.
In this work, we demonstrate that tipping point analysis of strain data can provide reactive and predictive indicators of cracking and structural transitions in a reinforced concrete system. The method is able to detect trend-driven transitions in a short time series of approximately 2000 datapoints, providing a clear indication of when a concrete beam under gradual bending progresses from a linear to a non-linear strain response. The method is also able to provide an early warning signal of the appearance of bifurcations, such as cracks, with a forewarning of 200-500 datapoints. The method, which was originally developed for applications in geophysics, shows promising results in the area of structural health monitoring, in particular, for real-time observations of civil constructions.
\end{abstract}




\section{Introduction}

Large-scale sensor networks for monitoring the health of civil megastructures are becoming increasingly common. As bridges and towers are now instrumented with hundreds of sensors $[1,2]$, their safe operation can be assured, provided the vast quantities of data can be managed. Indeed, the growing data deluge in structural health monitoring (SHM) will require new methods for rapid and automated data analysis, so that indicators of damage can be promptly investigated and acted upon [3].

The stages of damage identification in SHM include: 1) detection, 2) location and 3) assessment of the severity of damage. The first task is therefore not a choice of how to analyse the data, but where to start the analysis [4]. Various techniques for signal or statistical damage identification have been proposed to solve the detection problem: Hilbert-Huang transforms [5], independent component analysis [6] and Bayesian approaches [7] being the most common.

These techniques all aim to identify and locate damage (in time) so that more complex, model-based approaches can quantify the severity within a confined search space. The limitation of many of these methods is that they require estimates or prior knowledge of the system state before the damage has occurred. In the absence of data, this choice is at risk of being subjective and vagueł. Information on the system's behaviour after damage, while not strictly required, can also greatly improve damage detection accuracy. However, the main drawback of all of these methods is that they are not inherently predictive and can only detect damage once it has already occurred.

In the work presented here, we outline and apply the novel framework of 'tipping point analysis' to a reinforced concrete system to provide early warning indicators of transitions in structural behaviour before they occur. The tipping point framework, which consists of the degenerate fingerprinting method $[9$, $10,11]$ and potential analysis $[11,12,13]$, can also be used to detect damage indicators in historical data, and requires no knowledge of the system's state before or after damage [14]. The method has already been applied to large time series data, successfully detecting the increasing non-linearities which lead up to critical transitions in climatology [13], and

$\ddagger$ It should be noted that the choice of a prior state is less important for Bayesian approaches when the dataset is large [8]. structural temperature and tilt records [15]. Here, we apply the method to a relatively short time series of strain measurements, to attempt automatic detection of cracking and degradation of a reinforced concrete beam under three-point bending. By using a limited dataset of 2000 timepoints, we demonstrate that the technique could, in principle, be performed by onsite hardware in a real-time monitoring application. This could allow health monitoring to become more predictive than reactive.

This paper begins with a description of the theoretical background linking the mechanical interpretation of a concrete beam under three-point bending to its changes in potential energy state. This provides a framework for the introduction of the degenerate fingerprinting method and potential analysis in Section 2. The experimental and sensing set up for the threepoint bending test is described in Section 3 before the results and discussion are presented in Sections 4 and 5 respectively.

\section{Theoretical background}

In SHM applications, strain sensor noise is typically filtered out and used to define measurement uncertainty [16]. Cleansed strain signals are then interpreted from a mechanical perspective, so that damage can be estimated from the presence of features or from predefined material performance limits.

In this work, we demonstrate that if a structure is understood in terms of its potential energy states, then analysis of the strain noise itself can provide early-warning indicators of the occurence of damage. The method, alongside conventional approaches, allows transitions in structural behaviour to be identified and even predicted.

\subsection{Conventional mechanical approach}

Figure 1 illustrates how the performance of a singly reinforced concrete beam of length $L=60 \mathrm{~cm}$, width $b=10 \mathrm{~cm}$, and height $h=10 \mathrm{~cm}$, can be analysed during three-point bending. If the applied load, $F$, is small and does not induce stresses which exceed the modulus of concrete rupture, then the maximum tensile strain in the beam will be a linear function of the force applied [17].

If the strain in the tensioned surface of the concrete beam exceeds around $60 \mu \varepsilon$, then brittle 


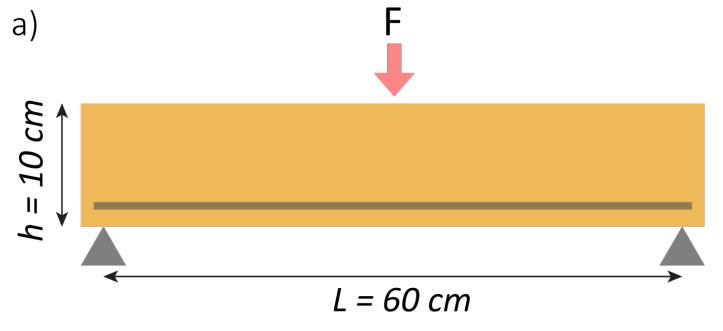

b)

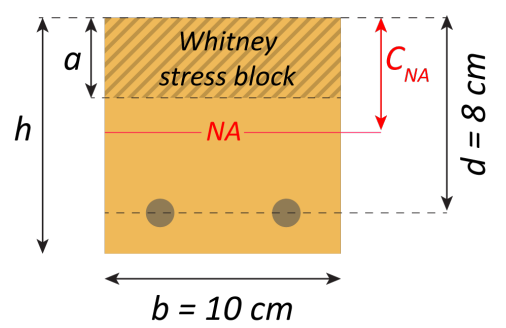

Figure 1. a) Side profile and b) cross-section of a single reinforced, simply supported concrete beam in three-point bending. The positions of the neutral axis (NA) and the Whitney stress block are highlighted.

fracture occurs and a distribution of cracks emerge [18]. The boundary layer below the steel reinforcement loses its strength, causing a shift in the neutral axis towards the compressive face, loss of beam stiffness and onset of non-linear behaviour. Under increasing loads, the reinforcement holds the concrete together until the steel begins to yield at a nominal load given by [19]:

$F_{f}=\frac{A_{s} \sigma_{y}}{L}\left(d-\frac{a}{2}\right)$,

where $A_{s}, \sigma_{y}$ and $d$ are the effective area, yield strength, and depth of the reinforcement respectively. The depth of the Whitney stress block is given by [20]:

$a=\frac{A_{s} \sigma_{y}}{0.85 \sigma_{c} b}=0.85 C_{N A}$,

where $C_{N A}$ is the depth of the neutral axis.

In this work, $\sigma_{c}=30 \mathrm{MPa}$ strength concrete is used with two $8 \mathrm{~mm}$ diameter steel rebars placed at depth $d=8 \mathrm{~cm}$. Substitution of steel yield strength, $\sigma_{y}$ $=500 \mathrm{MPa}$, suggests that the beam will completely fail at $F_{f}=12 \mathrm{kN}$. Assuming the strain transfer from the reinforcement to the concrete is perfect, the maximum strain in the tensile face of the concrete beam at failure will be:

$\varepsilon_{\max }=\frac{\sigma_{y}}{E_{s}}\left(\frac{h-C_{N A}}{d-C_{N A}}\right)$.

Substituting a steel stiffness of $E_{s}=200 \mathrm{GPa}$ reveals that $\varepsilon_{\max } \approx 3.5 \mathrm{~m} \varepsilon$.

\subsection{Energy state approach}

During three-point bending, the concrete beam can also be represented as a saddle-node bifurcation in a) uncracked

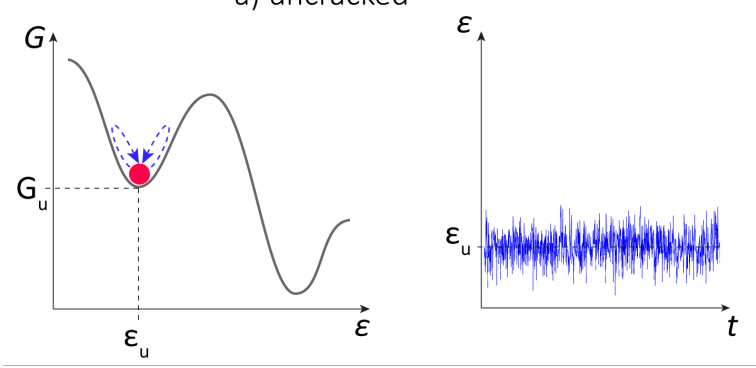

b) critical point
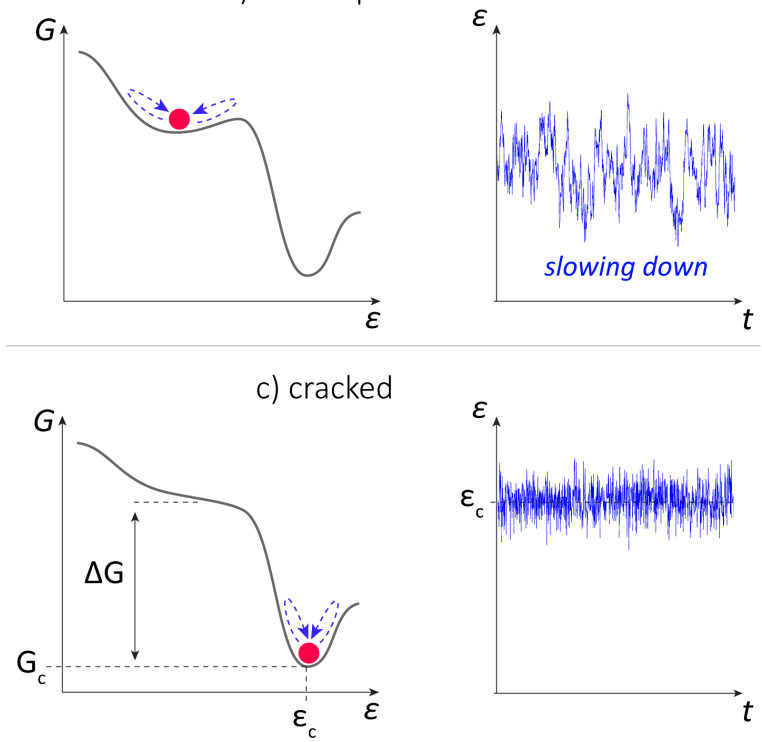

Figure 2. The strain energy $G$ as a function of the strain $\varepsilon$, for a concrete beam which is a) uncracked, b) at its critical point and c) cracked. The time-dependence of the strain signal at a constant force is also shown for each case.

energy-state space, as illustrated in Figure 2a. As shown, the undamaged, unloaded concrete beam is an elevated strain energy state with a local minimum (a so-called attractor) denoted $G_{u}$. Random perturbations, such as thermal or vibrational noise, induce small oscillations about the minimum, but the steep sides of the potential well cause the system to rapidly return to its equilibrium strain state, $\varepsilon_{u}$. Under these conditions, the decay rate, $\kappa$, of perturbations, has a large value. This results in rapid, uncorrelated oscillations in the measured strain noise (right panel of Fig.2a).

As the beam is loaded, the energy states begin to shift. Figure 2b represents a system which is at its tipping point: tensile stresses are nearing the magnitude required to cause a crack or some critical transition (otherwise known as a bifurcation). The sides of the potential well become shallower and so the system takes longer to leave and return to equilibrium. This manifests as a 'slowing down' of the system's response to perturbations: fluctuations change their 
dynamics as $\kappa$ decreases [10]. This behaviour is monitored using the degenerate fingerprinting technique described in Section 2.3.

Finally, as the transition occurs, there is a release of strain energy, $\Delta G$, as two new concrete surfaces are exposed to the air [21]. As shown in Figure 2c, the system irreversibly settles at some new strain state, $\varepsilon_{c}$, with a new local attractor, $G_{c}$. The strain noise again becomes rapid and uncorrelated until the next critical transition.

\subsection{Degenerate fingerprinting}

Degenerate fingerprinting is a tool for detecting the slowing down in noise which precedes many critical transitions $[9,10,11,15]$. Strain data, like that illustrated in Figure 2, can be described by a discrete time series of the form:

$\varepsilon_{r}[t]=\varepsilon_{o}[t]+\varepsilon_{n}[t]$,

where $\varepsilon_{o}[t]$ is the strain offset at each time point $t=$ $1,2, \ldots, M$. This offset is what conventional analyses aim to extract independently of Gaussian strain noise, $\varepsilon_{n}$. In degenerate fingerprinting, we instead extract either the raw strain data, $\varepsilon_{r}$, or the noise, $\varepsilon_{n}$, and consider it within a sliding window of length $N$. This yields a set of vectors:

$\overrightarrow{\varepsilon_{i}}=\varepsilon_{n \mid r}[i, \ldots, i+N-1]$

for $i=1, \ldots, M-N$.

Raw strain data, like that shown in Figure 6,tends to be non-stationary. When $\varepsilon_{r}$ is used as an input, the linear trend in each vector must be estimated and removed prior to continuing with the analysis. The method will then react to any 'trend-driven transitional tipping' - that is, transitions between linear and nonlinear behaviour in the overall strain response will be highlighted after they have occurred. Alternatively, the noise is extracted from $\varepsilon_{r}$ and input independently of all trends (in this work, we use wavelet denoising using a $4^{\text {th }}$ order Daubechies wavelet transform to extract $\varepsilon_{n}$ [22]). Using $\varepsilon_{n}$ in the analysis allows us to better detect bifurcational tipping, such as cracks, as these manifest themselves as a slowing down in strain fluctuations. As described in Section 2.2, the use of fluctuations is also predictive, as it allows the method to detect the approach to a critical transition.

To detect non-linearity or slowing down, the lag1 autocorrelation function (ACF) of each vector is calculated to obtain an 'ACF-indicator':

$c_{i}=\mathrm{ACF}^{1}\left\{\overrightarrow{\varepsilon_{i}}\right\}$.

Here, the $\mathrm{ACF}$ is defined:

$\mathrm{ACF}^{s}=\frac{\sum_{t=s+1}^{N}\left(\varepsilon_{t}-\bar{\varepsilon}\right)\left(\varepsilon_{t-s}-\bar{\varepsilon}\right)}{\sum_{t=1}^{N}\left(\varepsilon_{t}-\bar{\varepsilon}\right)^{2}}$ and lag- 1 autocorrelation is given for $s=1$. This indicator (which is normalised due to ACF properties) is a measure of how much each value of $\varepsilon_{i}[t]$ is dependent on its previous value, $\varepsilon_{i}[t-1]$. If the value of $c$ is plotted as the window slides along the series (note that $c$ is mapped to the end of the sliding window), then it will increase when non-linearity appears, or when critical slowing down creates trends in the noise. In this work, $c$ is estimated by fitting each $\overrightarrow{\varepsilon_{i}}$ to a lag-1 autoregressive AR(1) model of the form:

$y_{t}=c y_{t-1}+\eta_{t}$

where $\eta_{t}$ is Guassian white noise.

Note that, for noise fluctuations, the ACFindicator is also directly related to the time-step of the series, $\Delta t$, and to the decay rate of perturbations, $\kappa$ :

$c=e^{-\kappa \Delta t}$.

Therefore, when a bifurcation is being approached and the decay rate slows, $\kappa \rightarrow 0$ and $c \rightarrow 1$. Meanwhile, a flat and stable indicator suggests that there are no critical transitions in the time series [15].

\subsection{Potential analysis}

The tipping point framework allows one to analyse the number of potential wells in the dynamical system. Bifurcations in the time series are then visualised using a potential contour plot [13].

The double-well potential shown in Figure 2a can be parametrically described by a fourth order polynomial:

$G(\varepsilon)=a_{4} \varepsilon^{4}+a_{3} \varepsilon^{3}+a_{2} \varepsilon^{2}+a_{1} \varepsilon$.

The dynamic behaviour of the system is described by the pull, $G^{\prime}(\varepsilon)$, of the local attractor, added to random perturbations:

$\frac{d \varepsilon}{d t}=-G^{\prime}(\varepsilon)+\sigma \eta$

where the the stochastic component is described by unit variance Gaussian white noise, $\eta$, multiplied by some noise level, $\sigma$.

The probability of the system being at any given strain state, $\varepsilon$, at any given time, $t$, is described by the Fokker-Planck equation:

$\frac{\partial p}{\partial t}=\frac{\partial}{\partial \varepsilon}\left[G^{\prime}(\varepsilon) p\right]+\frac{\sigma^{2}}{2} \frac{\partial^{2} p}{\partial \varepsilon^{2}}$,

where $p=p(\varepsilon, t)$ is a probability density of the system's strain state. The stationary solution of equation (12) is [23]:

$p(\varepsilon) \sim \exp \left[-2 G(\varepsilon) / \sigma^{2}\right]$,

which can be rearranged to:

$G(\varepsilon)=-\frac{\sigma^{2}}{2} \log p_{d}(\varepsilon)$. 
Here $p_{d}$ is an empirically measurable probability density. The crucial outcome of equation (14) is that there is a one-to-one mapping between the number of modes in $p_{d}$ and the number of potential wells in $G$. This means that the number of potential wells can be estimated simply by analysing time-series data.

Detailed information on the method of the potential contour plot can found in [13], but essentially, the multiscale potential portrait is derived by estimation of the number of states based on the probability density function, which is performed in sliding windows of varying size. The number of wells detected is allocated to the time point on which the window is centered (i.e. the time point closest to $[\mathrm{i}+\mathrm{N}] / 2$ ). A potential contour plot (see Figure 7 for an example) is then generated, which maps time points against window size and allocates a colour to the number of wells detected, where 1: red, 2: green, 3 : cyan and 4: purple. The appearance of a vertical stripe of solid colour on a contour plot is strong evidence for a given number of potential wells, as it has been detected for all window sizes (along all time scales in the time series). If the vertical stripes in the plot transition from red to green, then this suggests that a second local potential well has been detected, and that bifurcation is about to occur.

\section{Experimental and sensor design}

To test the performance of the tipping point analysis during an application related to SHM, we monitored the strain and deflection of reinforced concrete beams while the force was controlled under three-point bending. Two beams (cross section $10 \mathrm{~cm} \times 10 \mathrm{~cm}$, length $110 \mathrm{~cm}$ ), were cast using a $30 \mathrm{MPa}$ strength concrete mix. To encourage cracking at the centroid of each beam, a greased acrylic card of width $2 \mathrm{~mm}$ was inserted $3 \mathrm{~cm}$ into the beams at their midsection during casting. The card was removed after 8 hours of curing, resulting in notched beams. Minimal reinforcement was provided on the notched side by two, internal, 8 $\mathrm{mm}$ diameter rebars. The beams were allowed to cure for 28 days prior to instrumentation and testing.

\subsection{FBG sensor design}

Optical fibre sensors are frequently used for SHM in civil engineering due to their high measurement performance, small size and multiplexability [24]. In this work, optical fibre Bragg grating (FBG) sensors were used to monitor surface strains in the tensioned face of the beam. FBG sensors are a $10 \mathrm{~mm}$ long periodic modulation in the refractive index of an optical fibre [25]. The gratings are illuminated using broadband light, guided within the optical fibre, and reflect a narrow distribution of wavelengths back
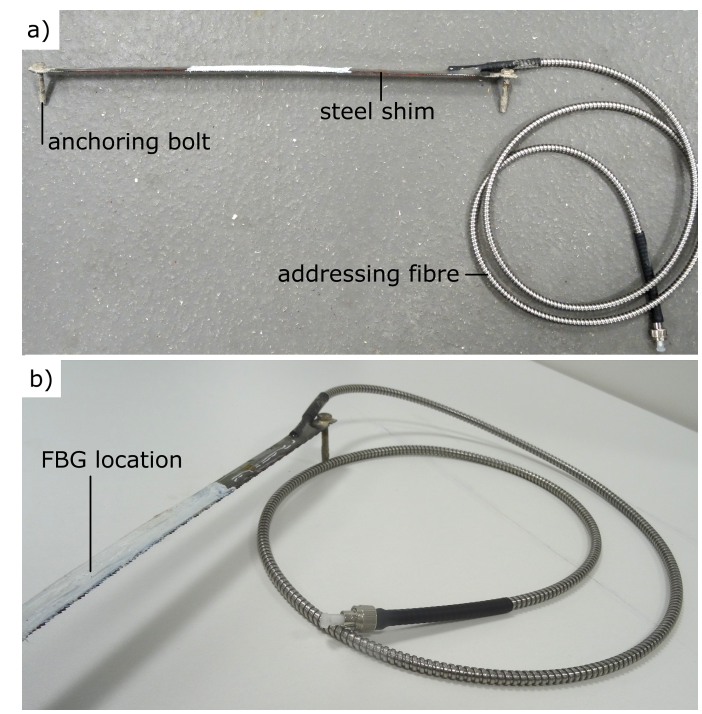

Figure 3. Photographs showing a) side profile and b) approximate FBG location of sensor housing construction. The sensor location is also coated in silicone sealant while the addressing fibre is protected by a flexible stainless steel conduit.

towards the light source. The centre of this wavelength distribution, termed the Bragg peak, $\lambda_{B}$, undergoes linear fractional shifts as strain, $\varepsilon_{s}$, and temperature changes, $\Delta T$, are applied to the FBG sensor:

$\frac{\Delta \lambda}{\lambda}=K_{\varepsilon} \varepsilon_{s}+K_{T} \Delta T$.

Here $K_{\varepsilon}$ and $K_{T}$ are the sensor's strain and temperature sensitivity respectively. Thermal and strain effects in equation (15) are decoupled by monitoring local temperatures using a second, adjacent FBG sensor which is isolated from strain (i.e. not bonded to the concrete beam).

In this work FBG strain sensors were epoxied to carbon-steel shim/bolt housing construction of length $L_{h}=30 \mathrm{~cm}$, shown in Figure 3. The $4 \mathrm{~mm}$ diameter bolts were inserted through holes in the shim and then bonded using induction brazing. Two $6 \mathrm{~mm}$ holes were drilled either side of the notch in the concrete beam and the bolts were grouted into these holes to affix the sensor (see Figure 4). FBGs at wavelengths between $1520 \mathrm{~nm}$ and $1580 \mathrm{~nm}$ were monitored via an armoured fibre connection, using a commercial optical interrogator at a rate of $2 \mathrm{~Hz}$ (strain resolution of 1 $\mu \varepsilon)$.

\subsection{Strain transfer}

Brazing, epoxying and grouting are all inexact manufacturing processes. Strains in the concrete, $\varepsilon_{c}$, are therefore not perfectly transferred to the sensor (i.e. the strain transfer, $\left.\alpha=\varepsilon_{s} / \varepsilon_{c}<1\right)$.

The FBG itself is a short-gauge sensor of $10 \mathrm{~mm}$ length, so it can effectively monitor the peak strain 
in the housing under bending. Meanwhile, the sensor housing is $L_{h}=30 \mathrm{~cm}$ long, and so its strain is described by:

$\varepsilon_{h}=\frac{\Delta L_{h}}{L_{h}}=\frac{u_{B}-u_{A}}{x_{B}-x_{A}}=\frac{1}{L} \int_{x_{A}}^{x_{B}} \varepsilon(x) d x+\frac{1}{L} \sum_{j} \Delta w_{j},(16)$

where $u_{A, B}$ are the displacements of the anchoring bolts, which are grouted into the concrete beam at locations $x_{A, B}$. The quantities $\Delta w_{j}$ are a set of added strain discontinuities caused by the concrete cracking [26]. The integral in equation (16) shows that the sensor housing provides an average measurement of the concrete strain between its two bonding points, resulting in a reduced strain and spatial resolution. This long-gauge sensing is, however, required in this case because concrete is an inhomogenous material. Monitoring over small length scales may provide measurements of single crack growth, but long-gauge sensors allow multiple cracks and bulk properties to be measured.

\subsection{Characterisation}

Prior to grouting, the temperature sensitivity of the sensor, shown in equation (15), was characterised using an environmental chamber so that ambient temperature fluctuations during testing could be accounted for. The FBG temperature sensitivity after epoxying to the steel housing was found to be $k_{T}=$ $16.2 \mathrm{ppm} /{ }^{\circ} \mathrm{C}$. This sensitivity is approximately 10 ppm $/{ }^{\circ} \mathrm{C}$ higher than that for an unbonded FBG [25]. The difference can be attributed to the added thermal expansion of the steel housing.

For this work, exact knowledge of the overall strain sensitivity was not essential. It could, however, be roughly estimated during three-point bending of the concrete beam by comparing the measured strains at concrete rupture and beam failure with the theoretical values derived in Section 2.1.

\subsection{Testing}

The instrumented beams were installed with sensors facing downwards, on simple supports placed $60 \mathrm{~cm}$ apart. Forces were applied to the top surface of the beam using a hydraulic force head. In the first experiment, an undamaged beam was loaded using a linear ramp rate of $0.0025 \mathrm{kN} / \mathrm{s}$ up until failure. The second beam, meanwhile, was cycled up to $5 \mathrm{kN}$ (equivalent to approximately $250 \mu \varepsilon$ measured strain) several times to simulate a damaged or aged structure. This 'damaged beam' was then force ramped to failure at a rate of $0.005 \mathrm{kN} / \mathrm{s}$.

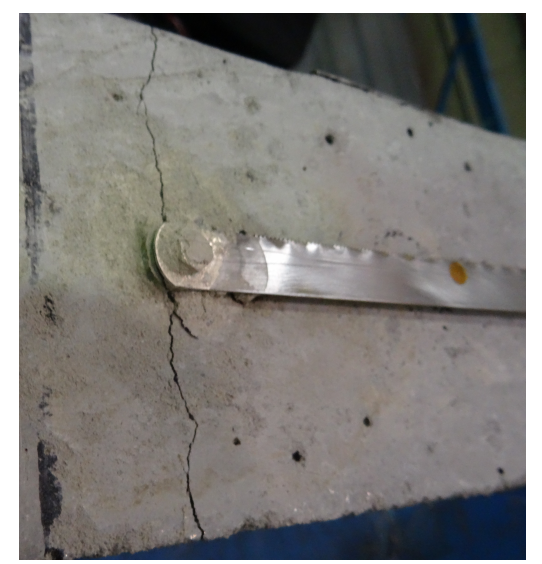

Figure 4. Photograph showing cracking of the beam at the grouted sensor anchorage.

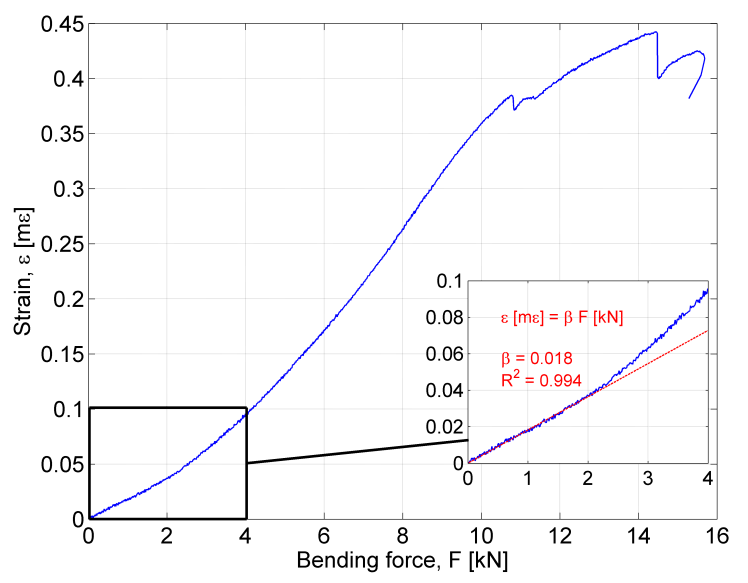

Figure 5. Measured strain in the undamaged concrete beam as a function of bending force. The linear strain portion is shown inset along with a linear fit.

\section{Results}

\subsection{Undamaged concrete beam}

4.1.1. Mechanical behaviour. Figure 5 shows the strain, measured using the FBG sensor, as a function of the bending force for the undamaged beam. As shown inset, the divergence from linear behaviour occurs at around $40 \mu \varepsilon$, which broadly agrees with the $60 \mu \varepsilon$ limit outlined in Section 2.1, assuming $60 \%$ strain transfer from the beam to the sensor. As the force is increased further, there are two competing effects:

- the build up of cracks at the centre of the beam causes the strain rate to increase: this is the dominant effect up to $6 \mathrm{kN}$ force;

- cracking at the sensor anchorages (shown in Figure 4) causes the strain transfer and strain rate to decrease: this is the dominant effect beyond $8 \mathrm{kN}$. 
Between $6 \mathrm{kN}$ and $8 \mathrm{kN}$ the strain rate is almost linear as the two competing effects balance.

Beyond $10 \mathrm{kN}$, sudden cracking at the sensor anchorages leads to discontinuous strain loss. The beam failed at $14 \mathrm{kN}$ at a measured strain of $0.45 \mathrm{~m} \varepsilon$. The force broadly agrees with equation (1), but the strain is lower than the $3.5 \mathrm{~m} \varepsilon$ predicted by equation (3). This suggests that cracking around the sensor anchorages has degraded the final strain transfer to around $10 \%$.

4.1.2. Strain data analysis. The top panel of Figure 6 shows the raw strain data, $\varepsilon_{r}$, as a time series (dashed line) and the corresponding ACF-indicator after linear detrending within the sliding window. Uncertainty in the indicator is calculated by varying the window length from $\frac{1}{4}$ to $\frac{3}{4}$ of the series length, and is shown by the shaded area around the curves. Beyond 500 seconds, the algorithm acquires enough time points to begin analysis and the indicator begins plotting. Between 600 and 800 seconds, the indicator remains reasonably flat as the strain response is linear. The onset of non-linear behaviour due to the decreasing strain transfer of the sensor is apparent as an increasing trend in the indicator at the point labelled 'A'. The sudden cracks cause the indicator to jump and then climb upwards at points labelled 'B' and 'C', showing that the indicator has reacted to the discontinuities.

The ACF-indicator after wavelet denoising is shown in the bottom panel of Figure 6 . The absolute value of the ACF-indicator is very low (in fact, negative, due to the effect of detrending), which shows the dynamics of the fluctuations contribute little to the tipping in the raw data. Nevertheless, upward trends at points labelled ' $\mathrm{D}$ ' and ' $\mathrm{E}$ ' are still visible, and these precede the discontinuities in the strain by $200-500$ datapoints. This demonstrates that the method can provide early-warning indications of changing system behaviour, even when the time series is short.

Potential contour plots for the raw strain data and fluctuations are shown in Figure 7 . The raw strain contour plot in Figure 7a shows some signs of vertical red bands after 1100 seconds and 1500 seconds due to the discontinuities of the crack, but the plot generally appears noisy. As was shown in [11], the patchy patterns in the plot indicate the absence of any global potential in the time series. This is expected, because the raw strain has a clear increasing trend. The contour plot for the fluctuations, shown in Figure 7b, is more promising. The plot is largely flat red (i.e., a one-well potential) but the analysis shows early stage green bands prior to 300 seconds, corresponding to a double-well potential. These bands precede the strain behaviour becoming non-linear after $40 \mu \varepsilon$, so may indicate the onset of early stage cracking
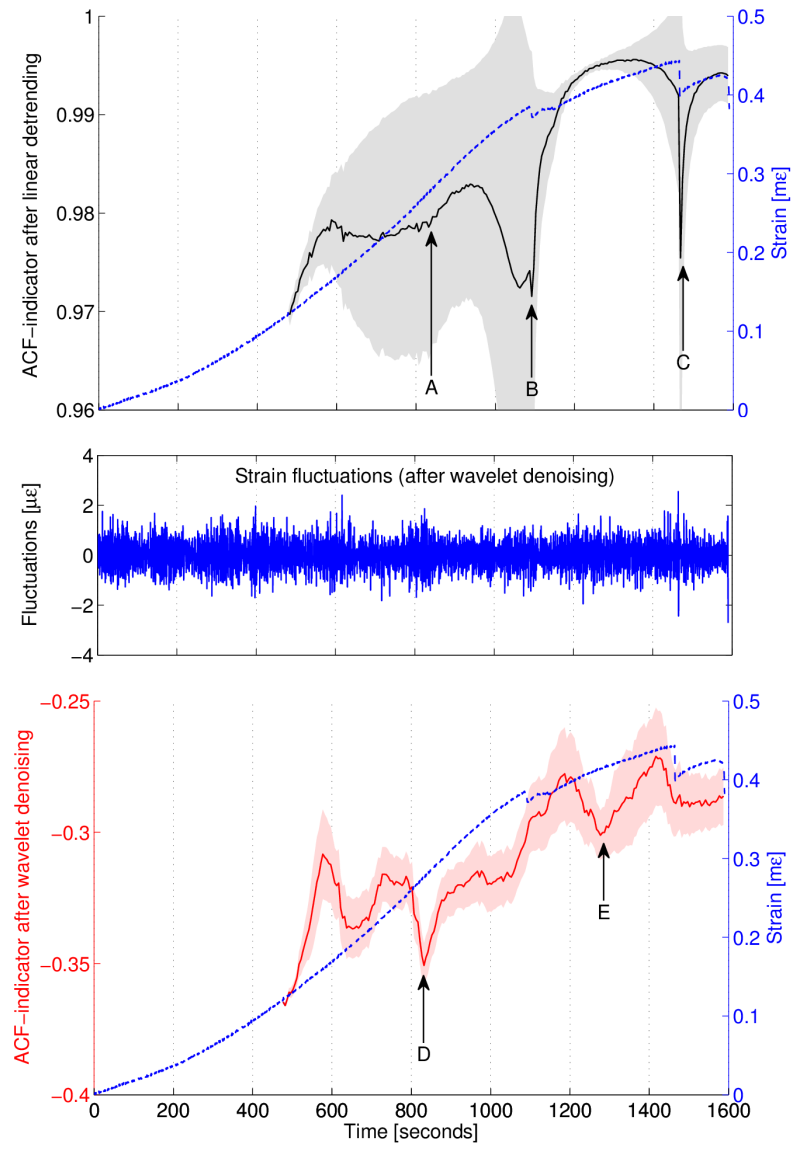

Figure 6. Strain analysis of the undamaged concrete beam, top panel: the ACF-indicator after linear detrending, middle panel: the strain fluctuations, $\varepsilon_{n}$, after wavelet denoising, bottom panel: the ACF-indicator after wavelet denoising. The strain data time series is also shown (dashed line) in the ACF-indicator plots for reference.

during concrete rupture. The other vertical green bands at around 500 seconds and 800 seconds precede the transitions between non-linear and linear strain behaviour. Other green bands may be indicative of bifrucations which are too minor to see in the data, or they could be false positives. Future work will aim to provide further thresholding and pattern recognition to objectively distinguish these.

4.1.3. Stroke data analysis. In real SHM applications, true displacement or deflection data for the structure is rarely available, and so conclusions must be drawn from strain data alone. However, in this beam test, data for the stroke of the force head could also be collected and analysed in the same manner as the strain data. The displacement data has a lower resolution and is less sensitive to small cracks, but provides a reliable measurement of the beam's global behaviour.

As shown in Figure 8, the ACF-indicator for the stroke after wavelet denoising generally increases 


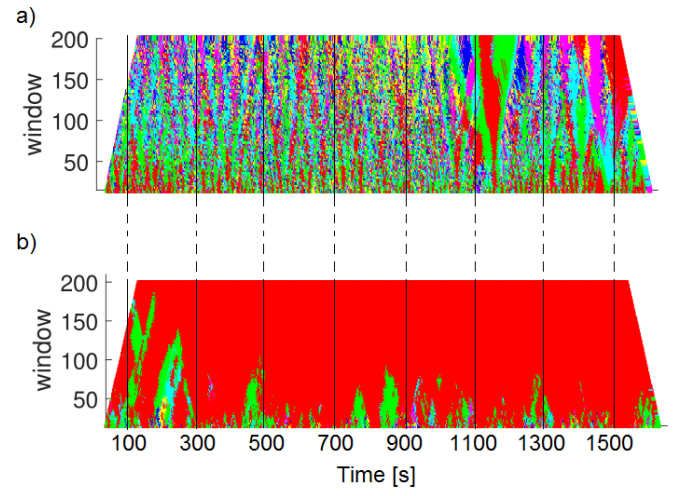

Figure 7. Potential contour plots for a) raw strain data and b) fluctuations after wavelet denoising for the undamaged concrete beam

throughout the test as the beam gradually reaches global failure. The indicator for the raw stroke data, meanwhile, appears to settle down at the point labelled 'A' as the stroke behaviour is linear at this point. The potential contour plots for the raw stroke data, shown in Figure 9a shows a solid red band at 800 seconds, while the contour plot for the flutuations in Figure 9b shows some double-well potential behaviour prior to this time. All of these behaviours suggest some change in material properties occurs at 800 seconds. Comparison with the strain data in Figure 6 reveals that this time matches up to the decreasing strain transfer regime of the strain sensor. This suggests that, at 800 seconds, the holes drilled in to the beam to install the sensor become a critical flaw. This is unsurprising given their depth and size, and is further confirmed by the fact that the beam failed at cracks which passed through the bolts.

\subsection{Damaged concrete beam (after cycling)}

4.2.1. Mechanical behaviour. Figure 10 shows the strain and stroke data for the damaged, pre-cycled beam as a function of the bending force. It is clear that the prior cycling has relaxed non-linearities below $250 \mu \varepsilon$, as cracking has already occurred. The stroke displacement reveals that the entire beam behaves nonlinearly after $6 \mathrm{kN}$, while the strain sensor behaves reasonably linearly up until beam failure. Again, this is thought to be due due to the balancing of cracking between and at the sensor bolt location. The beam failed at $13 \mathrm{kN}$, at strain of $650 \mu \varepsilon$, suggesting that the final strain transfer was $20 \%$.

4.2.2. Strain data analysis. Figure 11 demonstrates that, even though the loading rate of the beam is higher, the discontinuities in the strain at points labelled ' $A$ ' and ' $C$ ' are successfully picked up by the raw ACF-indicator after they occur. The indicator
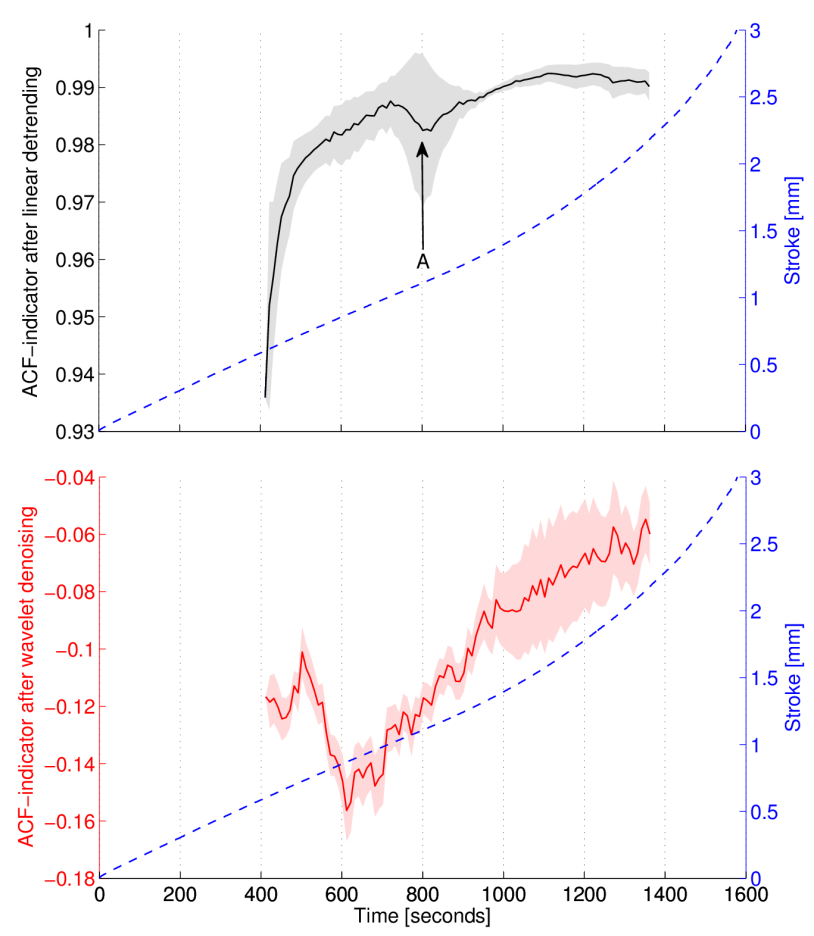

Figure 8. Stroke analysis of the undamaged concrete beam, top panel: the ACF-indicator after linear detrending, bottom panel: the ACF-indicator after wavelet denoising. The stroke time series is also shown (dashed line) for reference.

a)

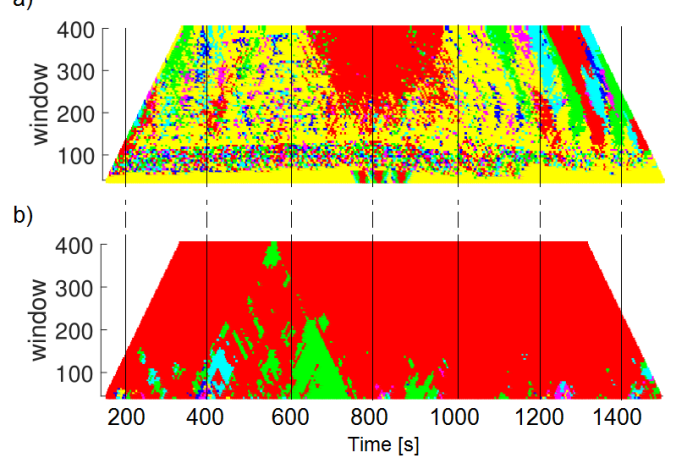

Figure 9. Potential contour plots for a) raw and b) fluctuation stroke data for undamaged beam.

does not pick up on the occurrence of the discontinuity at point ' $\mathrm{B}$ ' because it happens shortly after the discontinuity at point ' $A$ '. This demonstrates that the ACF-indicator needs more time to recover between events when the sliding window is large. Such close events may be monitored with small-size sliding windows.

Due to the higher speed of the force ramp in this test, the ACF indicator after wavelet denoising begins to climb before the crack at 1200 seconds (as shown by the point labelled ' $\mathrm{D}$ '), but does not have a chance to provide a reliable early warning indicator. Indeed, 


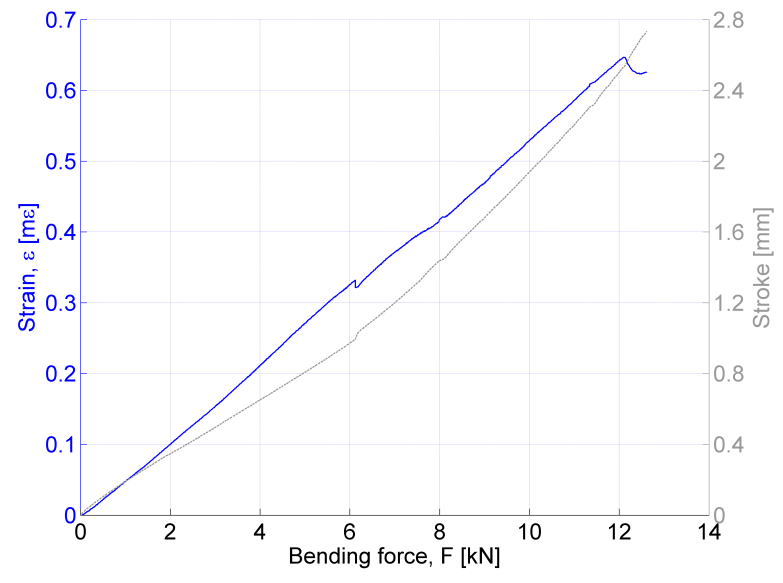

Figure 10. Measured strain and stroke in the damaged concrete beam as a function of bending force.
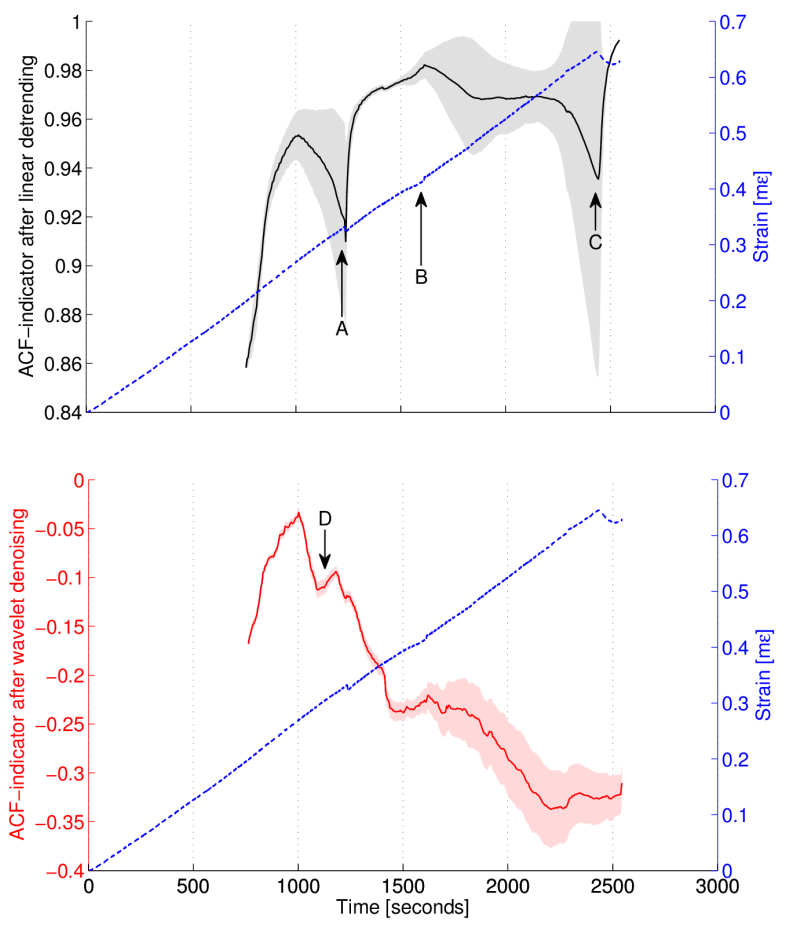

Figure 11. Strain analysis of the damaged concrete beam, top panel: the ACF-indicator after linear detrending, bottom panel: the ACF-indicator after wavelet denoising. The strain data time series is also shown (dashed line) in the ACF-indicator plots for reference.

the indicator after denoising generally fails to show any predictions, and this suggests that the changes in the system force are too rapid compared to the data acqusition rate. Note also that the upward step in strain at point ' $\mathrm{B}$ ', is due to a sudden jump in the force applied to the beam. This simulates a rapid, external impact - something that cannot be predicted.

\section{Discussion}

Degenerate fingerprinting and potential analysis have proven to be quick and highly adaptable methods for damage identification during a reinforced concrete monitoring application. However, tasks such as quantifying damage severity, tuning the algorithm's sensitivity and detecting false positives, still require an in-depth understanding of the behaviour of the structure, the sensor, and the interrogation system. The detection of cracking in the sensor anchorages highlights that the method does not discriminate between failure in the concrete beam, and failure in the sensor. While both events are arguably important, non-contact sensing methods, such as imaging, could be used to circumvent this issue in future work. However, a reasonable imaging stability would need to be demonstrated to prevent spurious indicators of damage.

The method's use of a sliding window means that a given number of time points are required before the ACF-indicator is generated. This caused some loss of early-stage strain behaviour in the work presented here. However, during continuous monitoring of a structure, only a minimal portion of data would be lost. In this work, we also chose to apply the method to a system under time-varying loads, but it could equally be applied to detect ageing in structural systems under more stationary conditions. The method is also transferable to other structural loads, such as temperature, as demonstrated in previous work [15].

A key limitation of degenerate fingerpinting is that the early warning indicators cannot detect abrupt bifurcations or those caused by sudden external variables such as impacts. For an increasing trend in the ACF-indicator to become apparent, bifurcational transitions should be gradual and accompanied by memory change (a slowing down in system noise). Fortunately, these limitations do not exclude the method's use in real-world applications, as many civil structures are relatively static. Even if the strain transitions are rapid compared to the interrogation rate, reactive monitoring to trend-driven transitions (using raw strain data) is still possible. If predictive monitoring for dynamically loaded structures is required, then this could be achieved by deploying high-speed measurement systems with $\sim \mathrm{kHz}$ interrogation rates [27]. In this work, a reasonably low interrogation rate was chosen to demonstrate that the algorithm could work without contributing further to data management problems.

The low computational cost of the tipping point analysis makes it highly suited to real-time monitoring. Furthermore, if the trends in the indicator could be classified automatically, then the sensor's communication network would only require 
higher rates of data streaming when nearing a critical transition. This would be particularly useful in wireless sensor networks, as data communication represents a significant portion of power consumption [28].

\section{Conclusion}

Comparative tipping-point analyses of raw strain data can provide information about the dominating influence of trend-driven transitional tipping in structural systems. Analysis of the strain fluctuations after wavelet denoising can also provide an early warning indicator of system bifurcations, such as cracking, with a forewarning of 200-500 datapoints. These methods could be applied to the real-time monitoring of structures which are experiencing various loads, and could facilitate both reactive and predictive damage detection in structural health monitoring.

\section{References}

[1] Cho S, Spencer B F, Jo H, Li J and Kim R E 2012 SPIE Newsroom

[2] Ni Y Q, Xia Y, Liao W Y and Ko J M 2009 Struct. Control Health Monit. 16 73-98

[3] Porter J H, Hanson P C and Lin C C 2012 Trends Ecol. Evol. 27 121-129

[4] Yang Y and Nagarajaiah S 2014 Mech. Syst. Signal Pr. 45 $1-23$

[5] Yang J N, Lei Y, Lin S and Huang N 2004 J. Eng. Mech. $13085-95$

[6] Zang C, Friswell M I and Imregun M 2004 Struct. Health Monit. 3 69-83

[7] Vanik M, Beck J and Au S 2000 J. Eng. Mech. 126 738-745

[8] Bernardo J and Smith A 2000 Bayesian Theory 1st ed (Wiley) ISBN 978-0-471-49464-5

[9] Held H and Kleinen T 2004 Geophys. Res. Lett. 31 L23207

[10] Livina V N and Lenton T M 2007 Geophys. Res. Lett. 34 L03712

[11] Livina V N, Ditlevsen P D and Lenton T M 2012 Phys. A 391 485-496

[12] Kwasniok F and Lohmann G 2009 Phys. Rev. E 80066104

[13] Livina V N, Kwasniok F and Lenton T M 2010 Clim. Past $677-82$

[14] Livina V N, Martins T M V and Forbes A B 2015 Chaos Interdiscip. J. Nonlinear Sci. 25036403

[15] Livina V, Barton E and Forbes A 2013 J. Civil Struct. Health Monit. 4 91-98

[16] Worden K, Farrar C R, Manson G and Park G 2007 Proc. R. Soc. Lond. Math. Phys. Eng. Sci. 463 1639-1664

[17] Bazant Z and Li Z 1995 J. Struct. Eng. 121 739-746

[18] Hwang L and Rizkalla S 1983 Effective tensile stress-strain characteristics for reinforced concrete Proceedings of the Canadian Society of Civil Engineering pp 129-147

[19] American Concrete Institute 2005 ACI 318

[20] Whitney C S 1937 ACI J. Proc. 33 483-498

[21] Anderson T 2005 Fracture mechanics: fundamentals and applications (CRC press)

[22] Addison P S 2002 The Illustrated Wavelet Transform Handbook: Introductory Theory and Applications in Science, Engineering, Medicine and Finance (CRC Press) ISBN 978-1-4200-3339-7
[23] Gardiner C W 2004 Handbook of Stochastic Methods: for Physics, Chemistry and the Natural Sciences 3rd ed (Springer) ISBN 978-3-540-20882-2

[24] Leung C K Y, Wan K T, Inaudi D, Bao X, Habel W, Zhou Z, Ou J, Ghandehari M, Wu H C and Imai M 2013 Mater Struct 48 871-906

[25] Hill K O and Meltz G 1997 J. Light. Technol. 15 1263-1276

[26] Glisic B 2011 Meas. Sci. Technol. 22035206

[27] Perry M, Orr P, Niewczas P and Johnston M 2013 J. Light. Technol. 31 2897-2903

[28] Popovici E, Magno M and Marinkovic S 2013 Power management techniques for wireless sensor networks: A review 5th IEEE IWASI pp 194-198 